\title{
Blasts Under 20,000 in Peripheral Blood White Cells
}

National Cancer Institute

\section{Source}

National Cancer Institute. Blasts Under 20,000 in Peripheral Blood White Cells. NCI

Thesaurus. Code C148071.

A quantitative microscopic finding indicating that less than 20,000 of the total number of nucleated cells in a peripheral leukocyte sample are immature mononuclear cells. 